\title{
Prediction of the haemodynamic response to tracheal intubation: comparison of laser-Doppler skin vasomotor reflex and pulse wave reflex
}

\author{
M. Luginbühl ${ }^{1}$, F. Reichlin ${ }^{2}$, G. H. Sigurdsson ${ }^{4}$, A. M. Zbinden ${ }^{3}$ and S. Petersen-Felix ${ }^{1}$ \\ ${ }^{1}$ Staff Anaesthesiologist, Department of Anaesthesiology University Hospital of Bern, CH-3010 Bern, \\ Switzerland. ${ }^{2}$ Department of Anaesthesiology, Kantonsspital Münsterlingen, Switzerland. \\ ${ }^{3}$ Research Section, Department of Anaesthesiology University Hospital of Bern, Switzerland. \\ ${ }^{4}$ Department of Anaesthesiology and Intensive Care, University Hospital Reykjavik, Iceland
}

*Corresponding author

\begin{abstract}
Background. The laser-Doppler skin vasomotor reflex (SVmR) caused by tetanic stimulation of the ulnar nerve may be a test that can predict the haemodynamic response to tracheal intubation. A decrease in pulse wave amplitude (pulse wave reflex, PWR) may be an alternative index of this response. We compared the abilities of PWR and SVmR to predict the haemodynamic response to tracheal intubation and studied how alfentanil, muscle relaxation, stimulation site and stimulation pattern affected the two reflexes.
\end{abstract}

Methods. Anaesthesia was induced and maintained with $2 \%$ sevoflurane and $50 \%$ nitrous oxide in two groups of 10 ASA status I patients. Tetanic stimuli were applied to the flexor muscles of the forearm and the ulnar nerve before and after administration of vecuronium. The change in skin blood flow (laser-Doppler) and pulse wave amplitude (pulse oximetry) after a 5 and $10 \mathrm{~s}$ stimulation was measured on the opposite hand. If skin blood flow (laser-Doppler) decreased by more than $10 \%$, a computer-controlled infusion of alfentanil was started and the target plasma concentration was increased in steps until this response was suppressed $(<10 \%)$. The trachea was intubated and arterial pressure and heart rate responses were recorded. Plasma alfentanil concentration was measured.

Results. When PWR and SVmR were suppressed, the haemodynamic response to tracheal intubation was reduced in 100 and $53 \%$ of patients respectively. PWR and SVmR responses decreased with increasing plasma alfentanil concentration. The SVmR response to muscle stimulation was reduced by muscle relaxants. The pulse wave response to both muscle and neural stimulation was reduced by relaxants. The responses to 5 and $10 \mathrm{~s}$ stimulations were similar.

Conclusion. An absent SVmR does not predict a blunted arterial pressure or heart rate response to tracheal intubation. The PWR may be a better predictor.

BrJ Anaesth 2002: 89: 389-97

Keywords: intubation, tracheal; measurement techniques, laser-Doppler flowmetry; measurement techniques, pulse wave amplitude; pain, experimental

Accepted for publication: April 16, 2002

Changes in arterial pressure or heart rate caused by painful stimulation may be harmful in patients with cardiovascular disease. ${ }^{1}$ Tracheal intubation is a powerful noxious stimulus. A $50 \%$ greater anaesthetic concentration is needed to suppress movement after intubation than after skin incision. $^{2-4}$ The minimal alveolar concentration required to suppress the haemodynamic response to skin incision
(MAC bar) is 1.3 times MAC. ${ }^{5}$ Variation in anaesthetic drug requirement between patients means that haemodynamic responses to tracheal intubation and skin incision may be insufficiently reduced. A small painful stimulus and an appropriate method of assessing the response to this stimulus would be useful for assessing the haemodynamic reactivity of an individual patient to a greater stimulus. 
Shimoda and co-workers ${ }^{6}$ suggested that cutaneous vasoconstriction after electrical tetanic stimulation of the ulnar nerve (skin vasomotor reflex, SVmR) could predict the arterial pressure response to tracheal intubation. After suppressing the skin vasomotor reflex with sevoflurane, they observed a much reduced arterial pressure response to tracheal intubation. ${ }^{6}$ They used a $3 \mathrm{~s}, 40 \mathrm{~mA}, 50 \mathrm{~Hz}$ electrical stimulation of the ulnar nerve, ${ }^{6}$ which was different from the stimulus used by Z Zbinden and co-workers $\left(10 \mathrm{~s}, 60 \mathrm{~mA}, 50 \mathrm{~Hz}\right.$ to the forearm muscles). ${ }^{2}$ The effect of opioids and neuromuscular blockade on these responses was not assessed and the responses to muscle and neural stimulation were not compared.

Measurement of skin blood flow with laser-Doppler flowmetry is expensive and not used routinely in clinical practice. The amplitude of the pulse oximeter wave could be an alternative index of vascular tone ${ }^{7}$ and correlates with the laser-Doppler signal. ${ }^{8}$ We considered the possibility that measurement of pulse oximeter amplitude, which is widely available, might be as useful as the laser-Doppler method to indicate vasoconstriction. We measured the pulse wave reflex (PWR) as the minimal pulse wave amplitude after a painful stimulus divided by the mean pulse wave amplitude before the stimulus.

We set out to confirm that $S V m R$ could predict a reduced haemodynamic response to tracheal intubation. We used an opioid instead of sevoflurane and assessed the PWR as a predictor of the haemodynamic response to intubation. We also assessed the effect of plasma alfentanil concentration and compared the effects of muscle relaxation and the effects of muscle and ulnar nerve stimulation on the two reflex responses.

\section{Patients and methods}

\section{Patients}

After approval by the local ethics committee and after written informed consent, we studied two groups of 10 ASA physical status I patients having elective surgery under general anaesthesia. We excluded patients who could not give informed consent, those receiving treatment that could affect MAC (e.g. sedative or tricyclic antidepressant drugs) and patients with a difficult airway.

\section{Study plan}

Patients were given omeprazole $40 \mathrm{mg}$ (Antra ${ }^{\circledR}$; AstraZeneca, Zug, Switzerland) on the evening before the study. In the operating theatre, standard monitoring devices were applied (Datex AS3 Compact, Datex-Ohmeda; Instrumentarium Corporation, Helsinki, Finland), a venous cannula was inserted in the non-dominant arm, and patency of the ulnar artery of both arms was assessed with Allen's test. On the non-dominant hand, an angled laser-Doppler flowmeter probe (PF 404; Perimed, Järfälla, Sweden) was placed on the fourth finger, a pulse-oximeter probe on the third finger and a skin temperature probe on the palm. The patient was then covered with a warming blanket (Bair Hugger; Augustine Medical, MN, USA). When the laser-Doppler signal was stable, the patients were asked to perform a brisk inspiration (inspiratory gasp), which should cause a shortlasting skin vasoconstriction. ${ }^{9}$ Patients without this reaction were excluded from the study as this indicates abnormal vasomotor function. ${ }^{9}$

\section{First study}

Anaesthesia was induced in the first group of 10 patients by vital capacity inhalation of $7 \mathrm{vol} \%$ sevoflurane in nitrous oxide/oxygen (ratio 1:1), and the end-tidal sevoflurane and nitrous oxide concentrations were kept at 2 and $50 \mathrm{vol} \%$ respectively. The patients were ventilated by facemask and the end-tidal carbon dioxide concentration was maintained between 35 and $45 \mathrm{~mm} \mathrm{Hg}$ throughout the study. A 20-G radial arterial cannula was inserted in the non-dominant arm. A temperature probe was placed in the rectum.

Skin electrodes for electrical stimulation were placed along a line between a point $1 \mathrm{~cm}$ ulnar to the mid-point of the cubital fold (point $\mathrm{A}$ ) and a point $1.5 \mathrm{~cm}$ ulnar to the mid-point of the first wrist fold (point $\mathrm{B}$ ). The muscle electrodes were attached 5 and $13 \mathrm{~cm}$ distal to $A^{2}$ and the ulnar nerve electrodes 15 and $23 \mathrm{~cm}$ distal to A. ${ }^{6}$ A $5 \mathrm{~s}, 60$ $\mathrm{mA}, 50 \mathrm{~Hz}, 0.25 \mathrm{~ms}$ square-wave electrical stimulus ${ }^{10}$ was applied by a Digitimer DS 7 constant current stimulator with a Digitimer DG2 trigger generator (Digitimer, Welwyn Garden City, Hertfordshire, UK) and a timer device (constructed in our laboratory). The positive pole of the stimulator was attached proximally. The tetanic stimulation was always applied first to the muscles and then to the ulnar nerve. After $10 \mathrm{~min}$ of equilibration, the first tetanic stimulation was applied. The second stimulation followed 3-5 min after vecuronium $0.1 \mathrm{mg} \mathrm{kg}^{-1}$ i.v., when at least three of the four twitches of the train-of-four were suppressed. Muscle relaxation was maintained at this level by further doses of vecuronium $(0.02 \mathrm{mg}$ i.v.) until the study was completed. If a positive laser-Doppler skin vasomotor reflex SVmR was present on ulnar nerve stimulation, an infusion of alfentanil was started to give a target plasma concentration of $25 \mathrm{ng} \mathrm{ml}^{-1}$. A Harvard 22 infusion pump (Harvard Apparatus, South Natick, MA, USA) was controlled by an IBM-compatible laptop computer running the Stanpump program (S. L. Shafer, Anesthesiology Service (112A), PAVAMC, Palo Alto, CA, USA) using pharmacokinetic parameters for alfentanil. ${ }^{11}$ After each positive $\mathrm{SVmR}$, the alfentanil target concentration was increased by $25 \mathrm{ng} \mathrm{ml}^{-1}$ and the stimulations were repeated. As soon as the laser-Doppler SVmR was negative (for definition see below), tracheal intubation was performed by an experienced anaesthetist. The haemodynamic response to tracheal intubation was determined off-line from the heart rate computed from the ECG and the arterial pressure values (Datex AS3 compact, Datex-Ohmeda) recorded on a 
personal computer every $10 \mathrm{~s}$ (Labview version 5.0; National Instruments Corporation, Austin, TX, USA). An arterial pressure increase of more than $25 \mathrm{~mm} \mathrm{Hg}$ and a heart rate increase of more than 25 beats $\min ^{-1}$ were considered relevant. The mean arterial pressure and heart rate in the $120 \mathrm{~s}$ before intubation were used as reference. The duration of the procedure from the insertion of the laryngoscope to the inflation of the cuff and the view of the vocal cords according to Cormack and Lehane ${ }^{12}$ were recorded.

Before each stimulation and before intubation, arterial blood samples were taken to measure plasma alfentanil concentration. Arterial blood gas measurements were made before the first tetanic stimulation and before intubation. Ten minutes after tracheal intubation, data acquisition was stopped and anaesthesia continued according to clinical routine.

\section{Second study}

The second group of 10 patients took part in this study. A $10 \mathrm{~s}, 60 \mathrm{~mA}, 50 \mathrm{~Hz}, 0.25 \mathrm{~ms}$ square-wave electrical impulse was used. The target concentration of alfentanil was initially $50 \mathrm{ng} \mathrm{ml}^{-1}$ and was increased in steps of $50 \mathrm{ng} \mathrm{ml}^{-1}$. In all other aspects the plan was as in the first study.

\section{Laser-Doppler SVmR}

Blood flow in the finger was measured continuously with a laser-Doppler flow system (Periflux 4001; Perimed). The time constant of the flowmeter output amplifier was set to $0.02 \mathrm{~s}$. The raw signal of the laser-Doppler was digitized at $128 \mathrm{~Hz}$ (A/D conversion card; National Instruments Corporation) and stored on a computer, and was also displayed during the study (arbitrary perfusion units according to the manufacturer's specifications). The laserDoppler system was calibrated with a standard suspension of $2 \mathrm{~mm}$ latex spheres provided by the manufacturer (Perimed).

The mean laser-Doppler flow (perfusion units) was calculated at intervals of $1 \mathrm{~s}$ from the digitized raw signal. The skin vasomotor reflex amplitude (SVmR) was defined according to the equation

$$
\mathrm{SVmR}=1-(b / a)
$$

where $a$ is the mean laser-Doppler flow during the $120 \mathrm{~s}$ before stimulation and $b$ is the minimal laser-Doppler flow after stimulation plus the standard deviation of $a$. The reflex was calculated for every stimulation according to the monitor readings and was considered absent if $\mathrm{SVmR}$ was 0.1 or less (i.e. the blood flow decreased by $10 \%$ or less; modified from Shimoda and colleagues). ${ }^{6}$

\section{Pulse wave reflex}

The pulse wave of the pulse oximeter (Datex AS3 Compact, Datex-Ohmeda) was scaled in arbitrary units from -100 to
+100 and digitized at $128 \mathrm{~Hz}$, like the laser-Doppler signal. The beat-to-beat amplitude of the pulse wave was measured off-line using software developed in our laboratory. The PWR was computed in the same way using equation (1), with the laser-Doppler flow replaced by the pulse wave amplitude.

\section{Alfentanil plasma concentrations and performance of the target-controlled infusion}

Blood samples for alfentanil measurement were immediately centrifuged at $3500 \mathrm{~g}$ for $30 \mathrm{~min}$ and the plasma was stored at $-26^{\circ} \mathrm{C}$. Alfentanil plasma concentration was measured by gas chromatography-mass spectrometry ${ }^{13}$ with a detection limit of $0.04 \mathrm{ng} \mathrm{ml}^{-1}$. We found coefficients of variation of 2.2, 2.5 and $4.7 \%$ at concentrations of 200,50 and $5 \mathrm{ng} \mathrm{ml}^{-1}$ respectively for measurements made on the same day. Between days we found coefficients of variation of $4.2,6.8$ and $7.5 \%$ respectively. Fentanyl was used as internal standard.

\section{Data analysis and statistics}

The characteristics of the two groups were compared using Fisher's exact test and the Wilcoxon rank test as appropriate. Values are given as medians (first and third quartiles) or as mean (SD) if normally distributed.

\section{Anaesthetic drug concentrations}

From the measured alfentanil plasma concentrations of each subject, the prediction error of the pharmacokinetic model was calculated:

$$
P E=\frac{C_{\mathrm{m}}-C_{\mathrm{p}}}{C_{\mathrm{p}}} \times 100
$$

where $C_{\mathrm{m}}$ is the measured concentration and $C_{\mathrm{p}}$ is the predicted plasma alfentanil concentration. The median prediction error (MDPE; a measure of bias) and the median absolute prediction error (MDAPE=median $|\mathrm{PE}|$; a measure of accuracy) were computed for each individual. ${ }^{14}$ Subsequently, the means of the MDPE (MDPE) and the MDAPE (MDAPE) were calculated for the groups.

The mean end-tidal sevoflurane and nitrous oxide concentrations for the period from $60 \mathrm{~s}$ before to $60 \mathrm{~s}$ after each stimulation were determined for each subject. Subsequently, the mean coefficients of variation of the sevoflurane and nitrous oxide concentrations were determined for the study population.

\section{Prediction of response to tracheal intubation}

The responses to the last ulnar nerve stimulation before tracheal intubation were analysed to estimate the probability of reduced arterial pressure and heart rate response to tracheal intubation if the laser-Doppler SVmR and the PWR were absent, i.e. the negative predictive value of these tests. 
Effect of stimulation pattern on the reflex responses

The responses to stimulation before alfentanil administration in the first and second studies were compared (Mann-Whitney rank sum test) to detect a difference between the 5 and $10 \mathrm{~s}$ stimulations, considering muscle and ulnar nerve stimulation separately. As there was no difference between these responses, the results of the two studies were pooled for further analysis.

\section{Effect of plasma alfentanil concentration on the reflex responses}

The data were fitted to a non-linear regression model relating the laser-Doppler SVmR and PWR values to the corresponding measured plasma alfentanil concentrations according to the equation

$$
y=y_{0} \times e^{-a \times x}
$$

where $y$ is the reflex (either PWR or SVmR) at plasma alfentanil concentration $x, y_{0}$ is the reflex at zero alfentanil and $a$ is the regression coefficient. The inter-individual variation of the estimated parameters ( $y_{0}$ and $a$ ) was modelled with an additive error model.

$$
P_{\mathrm{i}}=P_{\mathrm{TV}}+\eta_{\mathrm{i}}
$$

where $P_{\mathrm{i}}$ is the parameter of the $i$ th individual, $P_{\mathrm{TV}}$ is the typical parameter value of the population, and $\eta_{i}$ is the random inter-individual variation of the parameter (with mean 0 and variance $\omega^{2}$ ).

The residual error of the predicted effect including intraindividual variation ${ }^{15}$ was also assumed to be additive:

$$
E_{\mathrm{ij}}=E_{\mathrm{TVi}}+\varepsilon_{\mathrm{ij}}
$$

where $E_{\mathrm{ij}}$ is the effect in the $i$ th individual at the $j$ th measurement, $E_{\mathrm{TVi}}$ the typical effect value for the $i$ th individual and $\varepsilon_{\mathrm{j}}$ the residual variation of the effect at the $j$ th measurement (with mean 0 and variance $\sigma^{2}$ ).

This non-linear mixed-effects model was implemented in Fortran pseudocode for use with the non-linear mixedeffects modelling program NONMEM. ${ }^{16}$ The NONMEM objective function was minus twice the logarithm of the likelihood (-2LL). The objective function was minimized to obtain the best estimation of the parameters of the structural model, $y_{0}$ and $a$, and the related parameters of the error models, $\eta$ and $\varepsilon$. The two parameters of the structural model were considered significant if the $95 \%$ confidence interval of the estimates did not include zero. The significance of each of the structural and error parameters was tested with the likelihood ratio test. A difference in the minimal value of the objective function between the reduced and the full model exceeding 3.841 was considered significant $(P<0.05)$. If the minimal value of the objective function did not differ significantly between the full and the reduced model, a parameter was eliminated from the model.

\section{Effect of muscle relaxation and stimulation site on the reflex responses}

A signed rank test was used to test for differences between neural and muscle stimulation (using the responses to stimuli before neuromuscular block) and to compare the responses to neural and muscle stimuli before and after injection of the muscle relaxant.

The statistical analysis, except for NONMEM, was performed with Sigmastat version 2.0 (Jandel Scientific Corporation, San Rafael, CA, USA). $P<0.05$ was taken as significant.

\section{Results}

\section{Patients}

Ten ASA I patients were enrolled in each of the two studies. The characteristics of the patients are summarized in Table 1. Except for a slight but significantly greater age of the patients in the second study, the two groups of patients were similar. All patients had a vasoconstriction response to inspiration. After induction of anaesthesia with sevoflurane, there was an increase in laser-Doppler flow (median (first and third quartiles)) in the finger from $34(24,96)$ to 193

\begin{tabular}{|c|c|c|}
\hline & $\begin{array}{l}\text { Study } 1 \text { (5 s stimulation) } \\
(n=10)\end{array}$ & $\begin{array}{l}\text { Study } 2 \text { (10 s stimulation) } \\
(n=10)\end{array}$ \\
\hline Gender (male/female) & $6 / 4$ & $7 / 3$ \\
\hline Age (yr): mean (range) & $25(20-37)$ & $33(21-51)$ \\
\hline Weight $(\mathrm{kg})$ : mean (SD) & $72(11)$ & $73(12)$ \\
\hline Height $(\mathrm{cm})$ : mean (SD) & $174(8)$ & $172(8)$ \\
\hline Mallampati score 1,2 & 8,2 & 5,5 \\
\hline Laryngoscopy score $1,2 *$ & 4,6 & 8,2 \\
\hline Duration of intubation (s): mean (SD) & $16.0(4.1)$ & $16.8(5.7)$ \\
\hline Type of surgery: ENT, other & $8,2^{+}$ & $8,2^{\ddagger}$ \\
\hline Arterial $\mathrm{PCO}_{2}(\mathrm{~mm} \mathrm{Hg})$ : mean (SD) & $42(5)$ & $40(9)$ \\
\hline Body temperature $\left({ }^{\circ} \mathrm{C}\right)$ : mean (SD) & $36.6(0.7)$ & $36.6(0.27)$ \\
\hline Skin temperature at stimulation $\left({ }^{\circ} \mathrm{C}\right)$ : mean (SD) & $36.0(0.7)$ & $35.7(0.8)$ \\
\hline
\end{tabular}

Table 1 Characteristics of the study population. *Laryngoscopy grading according to Cormack and Lehane. ${ }^{12}$ No patient had a Mallampati or laryngoscopy score of more than 2. Type of surgery other than ENT: "orthopaedic, ${ }^{\ddagger}$ abdominal 


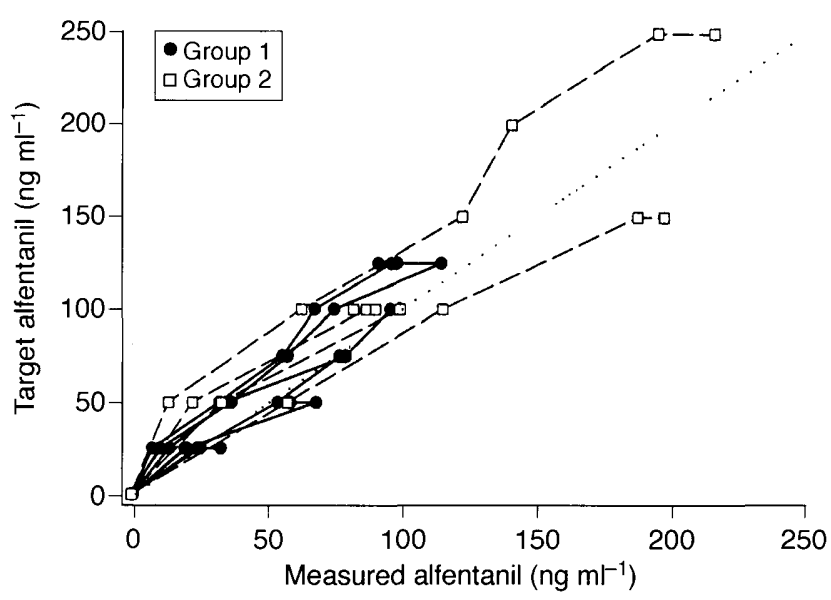

Fig 1 Measured and target plasma alfentanil concentrations at times of stimulation. The solid lines represent the subjects of study 1 , with one filled circle for each pair of stimuli (nerve and muscle). The dashed lines represent the subjects of study 2 , with one open square for each pair of stimuli. The last filled circle or open square of each line represents tracheal intubation. The dotted line is the line of equality.

$(157,245)$ perfusion units and in pulse wave amplitude (arbitrary units) from $2.0(1.5,2.6)$ to $8.5(4.6,10.1)$ (signed rank test, $P<0.001$ ). Finger temperature (mean (SD)) increased from $34.9(1.8)^{\circ} \mathrm{C}$ at the start to $36.2(0.9)^{\circ} \mathrm{C}$ at the end of the study procedure (signed rank test, $P<0.001$ ).

\section{Anaesthetic drug concentrations}

After equilibration, the end-tidal concentrations of nitrous oxide and sevoflurane were 49.02 (1.87) and 1.99 (0.07) vol\% respectively. The mean (range) coefficients of variation of the end-tidal concentration of nitrous oxide and sevoflurane were $2(0.2-7)$ and $0.5(0.1-5) \%$ respectively.

The MDPE, representing the bias of the alfentanil targetcontrolled infusion, and the MDAPE, representing its accuracy, were 7.6 and $17.7 \%$ respectively. Thus, the capacity of the selected pharmacokinetic parameters to predict plasma concentrations in our study population was comparable to that in a previous study (Fig. 1). ${ }^{11}$

Prediction of the haemodynamic reaction to tracheal intubation by $P W R$ and $S V m R$

The PWR before intubation was suppressed in seven of the 20 patients, whereas the laser-Doppler SVmR was suppressed in 19 of the 20 patients. One patient was intubated even though the SVmR remained positive at an alfentanil target concentration of $250 \mathrm{ng} \mathrm{ml}^{-1}$ as further prolongation of anaesthesia for the study was not appropriate. In the nine patients with a negative laser-Doppler SVmR but a positive PWR, the systolic arterial pressure is increased by more than $25 \mathrm{mmHg}$ and the heart rate by more than 25 beats $\min ^{-1}$ after tracheal intubation. Three of these were in the first and six in the second group of patients. This corresponds to a
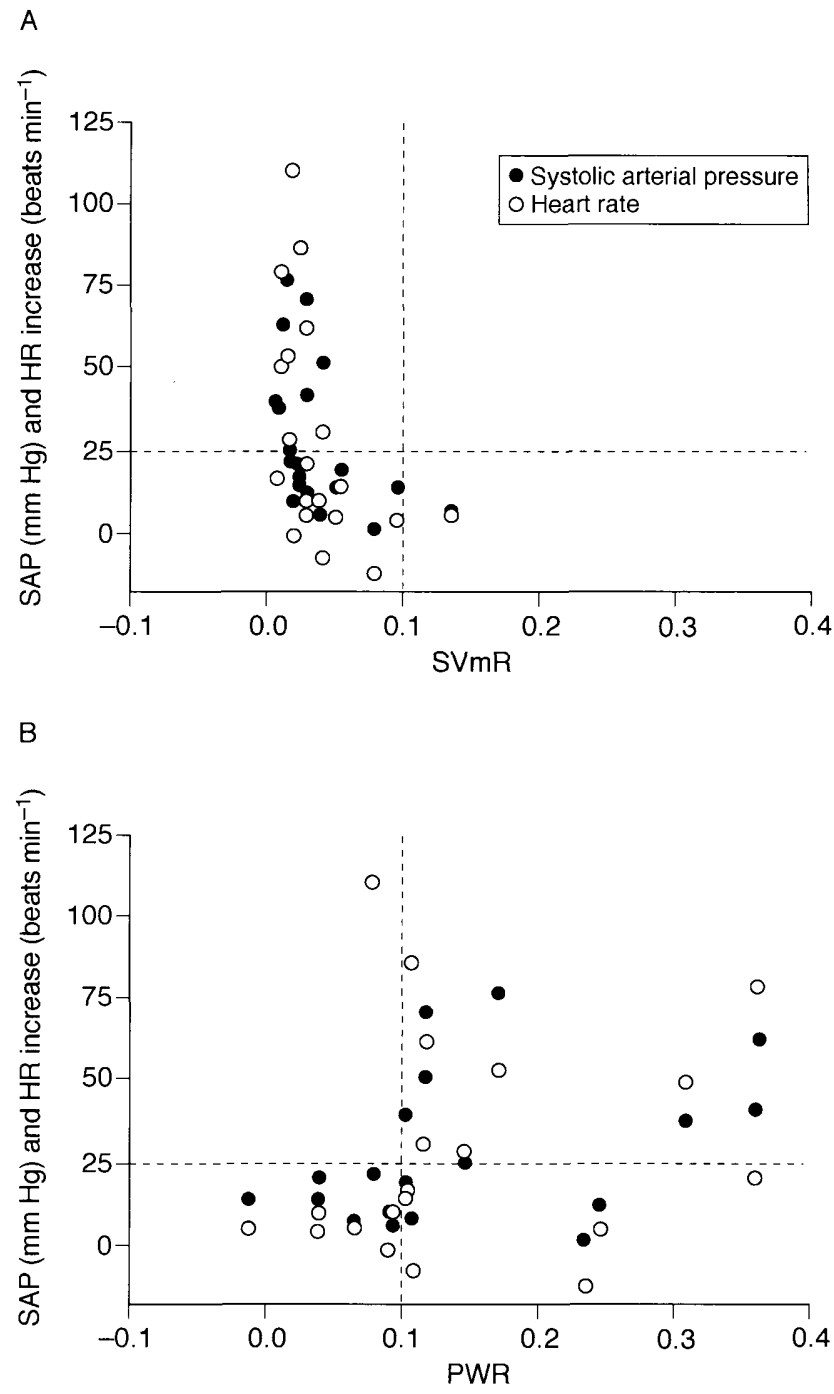

Fig 2 (A) The laser-Doppler skin vasomotor reflex (SVmR) and (B) the pulse wave reflex (PWR) related to the responses of systolic arterial pressure and heart rate to tracheal intubation. For SVmR and PWR a value below 0.1 was defined as no response (vertical dotted line). A systolic arterial pressure or heart rate increase at intubation was considered relevant if greater than $25 \mathrm{~mm} \mathrm{Hg}$ or 25 beats $\mathrm{min}^{-1}$ respectively (horizontal dotted line).

negative predictive value of $53 \%$ for laser-Doppler $\mathrm{SVmR}$ and $100 \%$ for the PWR (Fig. 2). The positive predictive value of the PWR was $69 \%$.

In seven of the nine patients with a false-negative $S V m R$, the SVmR was already negative before the alfentanil infusion was started. Intubation was therefore done with $50 \mathrm{vol} \%$ nitrous oxide and 2 vol\% sevoflurane. In the remaining two subjects a response occurred when the measured plasma alfentanil concentration was 98 and $81 \mathrm{ng} \mathrm{ml}^{-1}$ respectively. Only one patient with an absent laser-Doppler SVmR during inhalation anaesthesia ( 2 vol\% sevoflurane and 50 vol\% nitrous oxide) had no response to intubation. 


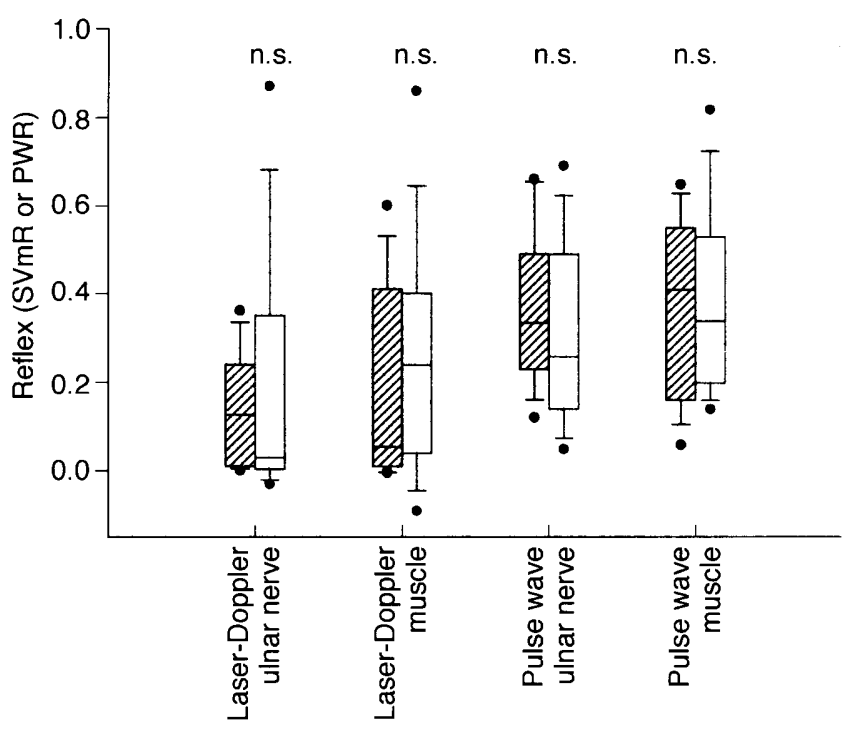

Fig 3 Laser-Doppler skin vasomotor reflex (SVmR) and pulse wave reflex (PWR) induced after tetanic stimulation of the ulnar nerve and of the flexor muscles of the forearm, before neuromuscular blockade and without opioids. The shaded bars represent $5 \mathrm{~s}$ stimuli and the open bars $10 \mathrm{~s}$ stimuli. Error bars represent 10 th and 90 th percentiles and points represent the 5th and the 95th percentiles.

\section{Effects of stimulus pattern, alfentanil concentration, muscle relaxation and site of stimulation}

The SVmR and PWR responses after 5 and $10 \mathrm{~s}$ stimuli given before alfentanil were similar (Fig. 3). The systolic arterial pressure increases after 5 and $10 \mathrm{~s}$ stimuli were 13.3 (6.6) $\mathrm{mm} \mathrm{Hg}$ (range 0-23) and 19.2 (12.1) $\mathrm{mm} \mathrm{Hg}$ (range 5.2-39) respectively (not significant).

The non-linear mixed effects model indicated a significant exponential decrease in both SVmR and PWR with increasing plasma alfentanil concentration (Fig. 4). The alfentanil effect was significant with ulnar nerve stimulation for both SVmR and PWR, whereas with muscle stimulation it was only significant for PWR. There was no significant difference between the slope parameters of PWR with ulnar nerve and muscle stimulation. The inter-individual error parameter $\eta_{\mathrm{y} 0}$ of the baseline reflex $\left(y_{0}\right)$ (equation (4)) showed considerable inter-individual variability, but the error parameter $\eta_{\mathrm{a}}$ of the regression coefficient $a$ did not. The parameter $\eta_{\mathrm{a}}$ therefore did not improve the fit and was excluded from the final model (Table 2).

Vecuronium $\left(0.1 \mathrm{mg} \mathrm{kg}^{-1}\right)$ reduced the laser-Doppler SVmR with direct muscle stimulation but not ulnar nerve stimulation, whereas the PWR was attenuated regardless of the stimulation site (Fig. 5). Neuromuscular blockade reduced the PWR after muscle stimulation more than after ulnar nerve stimulation $(P=0.04)$.

Muscle or ulnar nerve stimulation of either duration given before administration of neuromuscular blocking drugs induced a similar SVmR and PWR (Fig. 5). After administration of vecuronium, however, the SVmR was signifi- cantly less after muscle than after ulnar nerve stimulation, whereas PWR remained similar after stimulation at either site.

\section{Discussion}

In contrast to a previous study, ${ }^{6}$ we found that the laserDoppler SVmR does not reliably predict a relevant haemodynamic response to intubation. However, the PWR after electrical stimulation of the ulnar nerve predicted a haemodynamic response more reliably. Neuromuscular block attenuated but did not suppress the PWR after both muscle and ulnar nerve stimulation, whereas it attenuated the laser-Doppler SVmR after muscle stimulation.

A test of analgesia in the anaesthetized patient may help prevent unexpected haemodynamic reactions to severe stimuli. It would supplement anaesthetic depth monitors, such as the bispectral index and auditory evoked potential monitors, ${ }^{17} 18$ which indicate the hypnotic state. Although both SVmR and PWR were suppressed by alfentanil, the prediction of no response to tracheal intubation was poor with SVmR and good with PWR. The PWR, which could be easily measured clinically, could be a good alternative to the expensive and rarely available laser-Doppler (SVmR) technique. The cut-off value of 0.1 that has been suggested for SVmR, ${ }^{6}$ and which was also used for PWR in the present study, needs to be validated in a larger study.

From knowledge of the value for MAC intubation of sevoflurane in adults ${ }^{19}$ and the relationship between MAC and MAC bar, ${ }^{5}$ the responses after tracheal intubation found in patients not given alfentanil are unsurprising. They support the fact that haemodynamic responses to painful stimulation are not suppressed by volatile anaesthetics alone. $^{3}$

Because the response to tracheal intubation was badly predicted by laser-Doppler SVmR, we decided to increase the stimulus applied after the first 10 patients had been studied. The stronger stimulation did not affect the predictive value of the SVmR, however, and the responses (SVmR and PWR) were similar for the two stimulation patterns. Although the two patient groups were not randomized, they did not differ except for slightly greater age in the second group. If the same concentrations of sevoflurane and nitrous oxide are given to subjects of different age, the expected effect would be greater in the older subjects. ${ }^{20}$ In fact, the haemodynamic responses to tracheal intubation, but not the SVmR or PWR response, were significantly greater in the older subjects.

The ulnar nerve is the preferred site of stimulation to cause vasoconstriction. During neuromuscular blockade, PWR was inversely correlated with the measured alfentanil concentration regardless of the site of stimulation. Neuromuscular blockade did not suppress the PWR response a great deal and did not reduce its capacity to predict the response to tracheal intubation. 
A

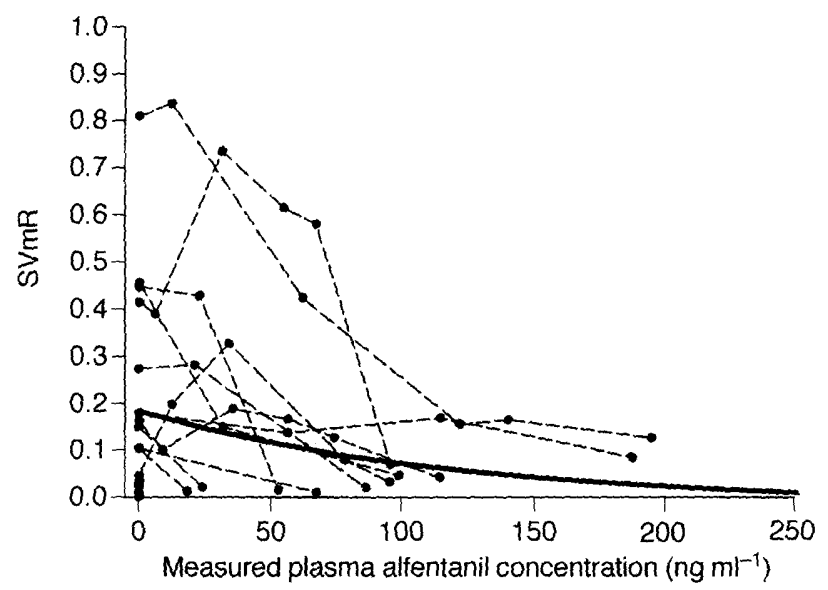

B

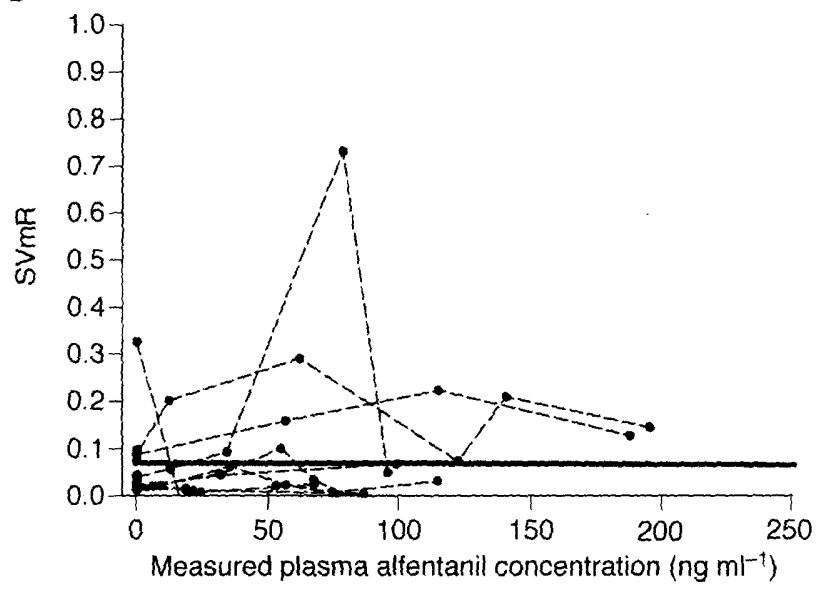

C

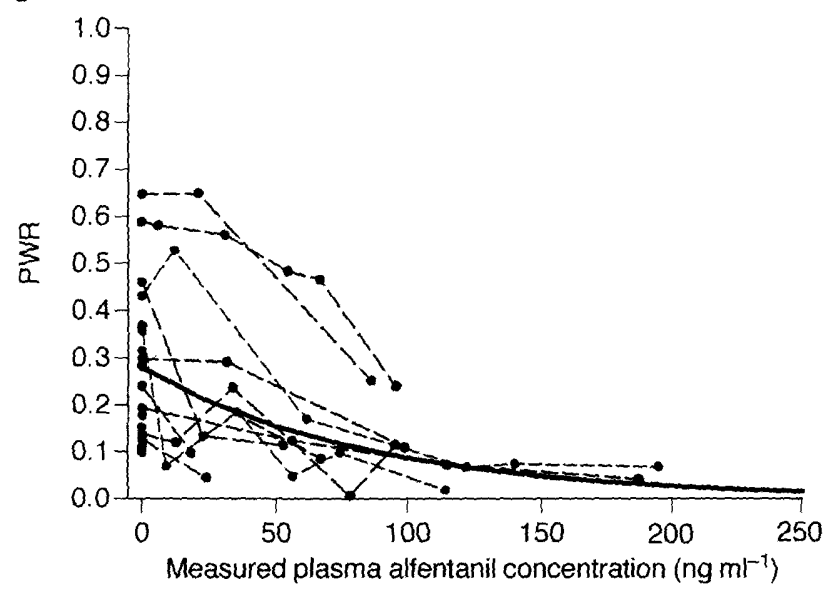

$D$

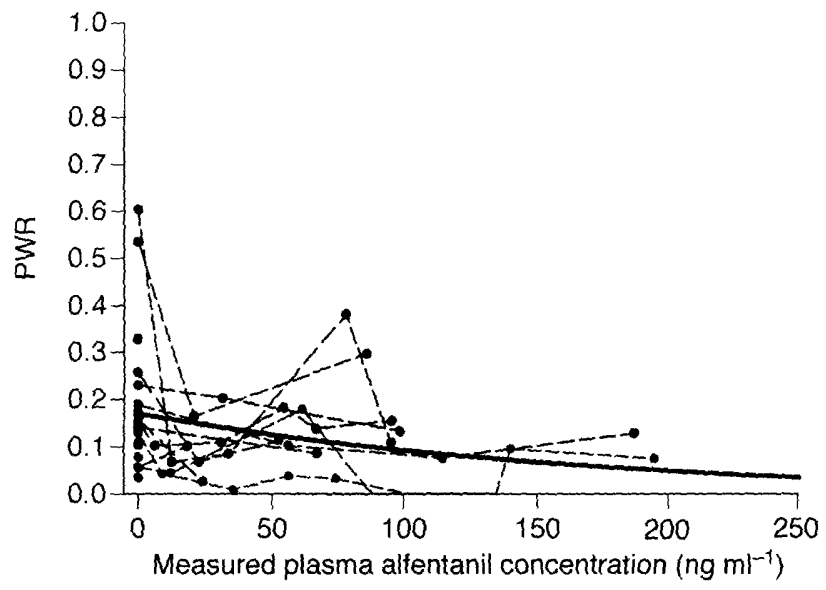

Fig 4 The laser-Doppler skin vasomotor reflex (SVmR; $A$ and B) and pulse wave reflex (PWR; C and D) recorded after ulnar nerve (A, C) and forearm muscle $(B, D)$ stimulation related to the corresponding measured alfentanil plasma concentrations. Data points from the same subject are linked by dashed lines. The straight-line indicates the best non-linear regression fit determined with NONMEM (Table 2).

Differences between our study and the previous study ${ }^{6}$ might partly explain the lower predictive value of laserDoppler SVmR in our study. Shimoda and co-workers ${ }^{6}$ carried out laryngoscopy and intubation 3-5 min after i.v. induction of anaesthesia in premedicated patients, but we stimulated our patients during pharmacological steady-state conditions. We applied the first stimulus a mean (range) of 22 (21-29) min after inhalation induction and intubated 50 min $(30-83)$ after induction. To prevent hypothermia, our patients were actively heated during the study, which increased skin temperature and induced vasodilation, shown by an increase in laser-Doppler flow and pulse wave amplitude before stimulation. Despite this vasodilation, tracheal intubation decreased pulse wave amplitude and finger blood flow (laser-Doppler). The decrease in pulse wave amplitude caused by tracheal intubation was correlated with the analgesic drug concentration, but the change in skin blood flow was not (data not shown). Because the $S V m R$ in response to nerve stimulation was negative in nine patients, they were intubated without alfentanil administration first. The increase in arterial pressure after tracheal intubation in these patients could have increased the superficial skin blood flow in the fingers and thus reduced SVmR, because the vasodilation induced by sevoflurane and skin warming may have prevented the transient skin vasoconstriction induced by intubation. In the 11 patients given alfentanil, however, the SVmR was not suppressed and the decrease in skin blood flow was still found. Considering the whole study population, neither PWR nor SVmR was correlated with the skin temperature, so heating and sevoflurane may have suppressed skin vasomotor reactions (laser-Doppler) only in some patients. PWR was not affected by temperature, which suggests it is a better measure than SVmR.

The laser-Doppler probe measures the microvascular blood flow in a small area of the fingertip to a depth of a few millimetres. The pulse oximeter probe, in contrast, measures the changing blood volume in the whole fingertip. Finger 
Table 2 PWR and SVmR and measured plasma alfentanil concentration (non-linear regression analysis). $y_{0}=$ baseline reflex before the start of alfentanil administration but after neuromuscular blockade; $a=$ non-linear regression coefficient; $\checkmark \sigma^{2}=\mathrm{SD}$ of the reflex in an individual subject (residual and intra-individual error), $-2 \mathrm{LL}=$ minimum value of NONMEM objective function according to the likelihood ratio test; $C V=$ coefficient of variation in the study population computed from the interindividual error $\eta$; PWR=pulse wave reflex; $S V m R=$ skin vasomotor reflex with site of stimulation in parentheses. The inter-individual variation of the baseline reflex $y_{0}$ was considerable; the inter-individual variation of the regression coefficient $a$ was small and did not improve the fit

\begin{tabular}{lllll}
\hline Reflex & $y_{0}$ & $\boldsymbol{a}$ & $\checkmark \sigma^{2}$ & $-\mathbf{2 L L}$ \\
\hline PWR ulnar nerve & 0.27 & 0.009 & 0.08 & -167.062 \\
SE & 0.04 & 0.002 & & \\
CV (\%) & 0.60 & - & & \\
PWR muscle & 0.17 & 0.006 & 0.11 & -164.423 \\
SE & 0.02 & 0.002 & & \\
CV (\%) & 0.39 & - & & \\
SVmR ulnar nerve & 0.19 & 0.008 & 0.13 & -126.649 \\
SE & 0.05 & 0.003 & & \\
CV $(\%)$ & 1.05 & - & & -163.724 \\
SVmR muscle & 0.07 & $3.96 \mathrm{E}-12$ & 0.12 & \\
SE & 0.02 & $9.18 \mathrm{E}-06$ & & \\
CV $(\%)$ & - & - & &
\end{tabular}

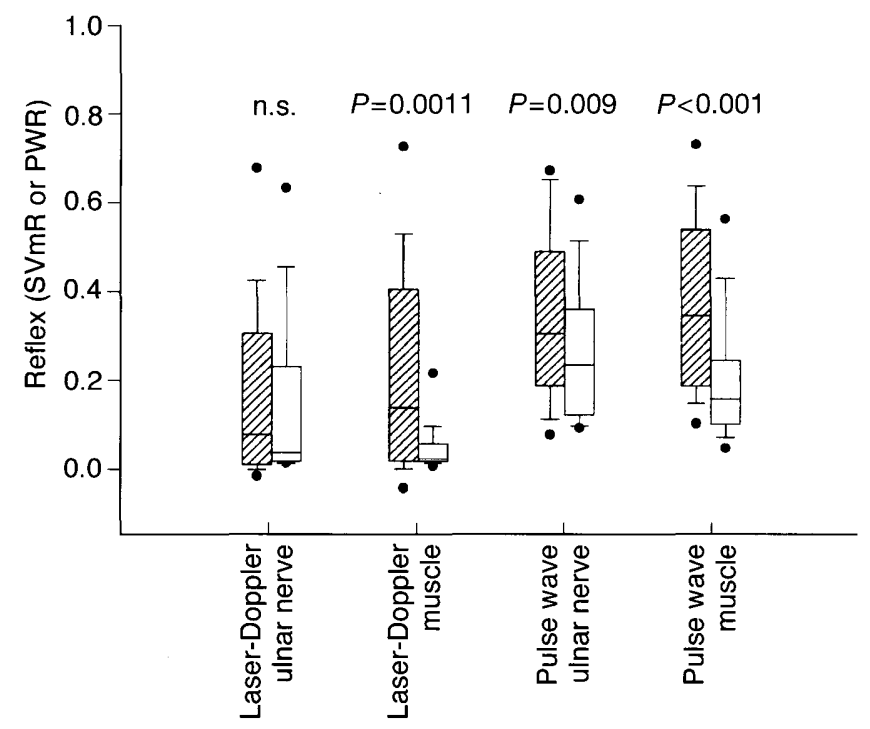

Fig 5 Laser-Doppler skin vasomotor reflex $(\mathrm{SVmR})$ and pulse wave reflex (PWR) after electrical tetanic stimulation of the ulnar nerve and the flexor muscles of the forearm. Twenty stimuli before (shaded bars) and 20 stimuli after (open bars) vecuronium $0.1 \mathrm{mg} \mathrm{kg}^{-1}$ in 20 patients anaesthetized with sevoflurane $(2 \mathrm{vol} \%)$ and nitrous oxide (50 vol\% in oxygen) without opioids. Box plots with error bars representing the 10th and 90 th percentiles and points the 5 th and 95 th percentiles.

blood flow is regulated mainly by local arteriovenous shunts. $^{21}$ Vasoconstriction to a cold stimulus in awake humans is more pronounced in deep finger vessels than in superficial ones. ${ }^{22}$ Vasoconstriction to pain in deep and superficial finger vessels has not yet been compared. Our data might suggest vasoconstriction in deep finger vessels is more pronounced than in superficial ones and is thus less suppressed by active warming of the body and volatile anaesthetics. This would explain how the PWR -in contrast to the laser-Doppler SVmR-was not attenuated in our study. Our results, in which acute sympathetic activation elicited by pain caused a transient vasoconstriction and thereby a change in the PWR, support previous data on angiotensin-induced vasoconstriction. ${ }^{23}$ Vasoconstriction with angiotensin slightly decreased the maximal photoplethysmography wave amplitude, by reducing the size of the early systolic component of the pulse wave, while the pulse pressure and the late systolic arterial pressure in the ascending aorta increased because the reflection wave increased. ${ }^{23}$

We conclude that a negative SVmR does not predict a reduced response of arterial pressure or heart rate to tracheal intubation. The PWR to a $5 \mathrm{~s}, 60 \mathrm{~mA}, 50 \mathrm{~Hz}$ tetanic impulse to the ulnar nerve could be a better predictor and warrants further study to confirm that the selected cut-off limit is appropriate and to determine the efficiency of PWR in predicting responses in patients of different ages and medical conditions.

\section{Acknowledgement}

This research was funded by the Department of Anaesthesiology, University Hospital of Bern, Switzerland.

\section{References}

I Mangano DT, Layug EL, Wallace A, Tateo I. Effect of atenolol on mortality and cardiovascular morbidity after noncardiac surgery. Multicenter Study of Perioperative Ischemia Research Group. N Engl J Med 1996; 335: 1713-20

2 Zbinden AM, Maggiorini M, Petersen-Felix S, Lauber R, Thomson DA, Minder CE. Anesthetic depth defined using multiple noxious stimuli during isoflurane/oxygen anesthesia. I. Motor reactions. Anesthesiology 1994; 80: 253-60

3 Zbinden AM, Petersen-Felix S, Thomson DA. Anesthetic depth defined using multiple noxious stimuli during isoflurane/oxygen anesthesia. II. Hemodynamic reactions. Anesthesiology 1994; 80: $26 \mid-7$

4 Inomata S, Watanabe S, Taguchi M, Okada M. End-tidal sevoflurane concentration for tracheal intubation and minimum alveolar concentration in pediatric patients. Anesthesiology 1994; 80: 93-6

5 Roizen MF, Horrigan RW, Frazer BM. Anesthetic doses blocking adrenergic (stress) and cardiovascular responses to incisionMAC BAR. Anesthesiology 1981; 54: 390-8

6 Shimoda O, Ikuta $Y$, Sakamoto M, Terasaki H. Skin vasomotor reflex predicts circulatory responses to laryngoscopy and intubation. Anesthesiology 1998; 88: 297-304

7 Shelley KH, Murray WB, Chang D. Arterial-pulse oximetry loops: a new method of monitoring vascular tone. J Clin Monit 1997; 13: 223-8

8 Shelley KH, Stout B, Silverman D. The pulse oximeter waveform compared to the laser Doppler waveform. J Clin Monit Comput 1999; 15: 247-8

9 Low PA, Neumann C, Dyck PJ, Fealey RD, Tuck RR. Evaluation of 
skin vasomotor reflexes by using laser Doppler velocimetry. Mayo Clin Proc 1983; 58: 583-92

10 Petersen-Felix S, Zbinden AM, Fischer M, Thomson DA. Isoflurane minimum alveolar concentration decreases during anesthesia and surgery. Anesthesiology 1993; 79: 959-65

II Raemer DB, Buschman A, Varvel JR, et al. The prospective use of population pharmacokinetics in a computer-driven infusion system for alfentanil. Anesthesiology 1990; 73: 66-72

12 Cormack RS, Lehane J. Difficult tracheal intubation in obstetrics. Anaesthesia 1984; 39: I 105-1 I

I3 Kingsbury DP, Makowski GS, Stone JA. Quantitative analysis of fentanyl in pharmaceutical preparations by gas chromatographymass spectrometry. J Anal Toxicol 1995; 19: 27-30

14 Varvel JR, Donoho DL, Shafer SL. Measuring the predictive performance of computer-controlled infusion pumps. J Pharmacokinet Biopharm 1992; 20: 63-94

15 Karlsson MO, Sheiner LB. The importance of modeling interoccasion variability in population pharmacokinetic analyses. J Pharmacokinet Biopharm 1993; 21 : 735-50

16 Sheiner LB, Beal SL. NONMEM User's Guide 1994. San Francisco (CA): University of California San Francisco, 1994
17 Rampil IJ. A primer for EEG signal processing in anesthesia. Anesthesiology 1998; 89: 980-1002

18 Dutton RC, Smith WD, Rampil IJ, Chortkoff BS, Eger El. Fortyhertz midlatency auditory evoked potential activity predicts wakeful response during desflurane and propofol anesthesia in volunteers. Anesthesiology 1999; 91 : 1209-20

19 Katoh T, Nakajima Y, Moriwaki G, et al. Sevoflurane requirements for tracheal intubation with and without fentanyl. Br J Anaesth 1999; 82: 56I-5

20 Mapleson WW. Effect of age on MAC in humans: a meta-analysis. $\mathrm{Br}$ J Anaesth 1996; 76: 179-85

21 Sessler DI, McGuire J, Hynson J, Moayeri A, Heier T. Thermoregulatory vasoconstriction during isoflurane anesthesia minimally decreases cutaneous heat loss. Anesthesiology 1992; 76: 670-5

22 Richardson D, Tyra J, McCray A. Attenuation of the cutaneous vasoconstrictor response to cold in elderly men. J Gerontol 1992; 47: $M 21 I-M 2 \mid 4$

23 Takazawa K, Tanaka N, Fujita M, et al. Assessment of vasoactive agents and vascular aging by the second derivative of photoplethysmogram waveform. Hypertension 1998; 32: 365-70 